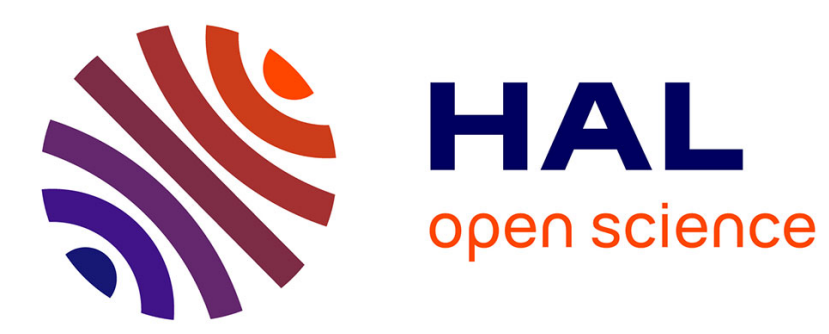

\title{
Effet protecteur de la néoglucogenèse intestinale sur le développement de l'obésité et du diabète
}

\author{
M. Soty, J. Dehedin, M. Raffin, G. Mithieux
}

\section{To cite this version:}

M. Soty, J. Dehedin, M. Raffin, G. Mithieux. Effet protecteur de la néoglucogenèse intestinale sur le développement de l'obésité et du diabète. Annales d'Endocrinologie, 2016, 77 (4), pp.293. 10.1016/j.ando.2016.07.905 . inserm-02393733

\section{HAL Id: inserm-02393733 https://www.hal.inserm.fr/inserm-02393733}

Submitted on 4 Dec 2019

HAL is a multi-disciplinary open access archive for the deposit and dissemination of scientific research documents, whether they are published or not. The documents may come from teaching and research institutions in France or abroad, or from public or private research centers.
L'archive ouverte pluridisciplinaire HAL, est destinée au dépôt et à la diffusion de documents scientifiques de niveau recherche, publiés ou non, émanant des établissements d'enseignement et de recherche français ou étrangers, des laboratoires publics ou privés. 


\section{ScienceDirect}

www.sciencedirect.com www.em-consulte.com
Annales d'Endocrinologie

Annales d'Endocrinologie 77 (2016) 293-295

SFE Bordeaux 2016

Communications orales et présentations orales de poster

\section{Métabolisme et insulino-résistance ${ }^{\text {th }}$}

\section{CO-061 \\ Effet protecteur de la néoglucogenèse intestinale sur le développement de l'obésité et du diabète \\ M. Soty (Dr)*, J. Dehedin (Mlle), M. Raffin (Mlle), G. Mithieux (Dr) Inserm U1213/UCBL, Lyon, France \\ * Auteur correspondant. \\ Adresse e-mail : maud.soty@gmail.com (M. Soty)}

La production de glucose par l'intestin (PIG) induit un signal nerveux au niveau de la veine porte (signal glucose portal) capable d'exercer des effets bénéfiques sur l'homéostasie énergétique et glucidique. L'induction de la PIG est impliquée dans l'effet de satiété des protéines ou dans les améliorations métaboliques à la suite d'un by-pass gastrique. Indépendamment de toute modification nutritionnelle, nous avons étudié le rôle de la PIG sur le développement de l'obésité et du diabète.

Nous avons généré des souris sur-exprimant le gène de l'unité catalytique de la glucose-6-phosphatase (enzyme clef de la néoglucogenèse) spécifiquement au niveau de l'intestin par une approche Rosa26-CRE-Lox (souris SIC) et nous avons étudié leur métabolisme glucidique et énergétique nourries en régime High Fat High Sucrose (HF/HS).

Les SIC prennent significativement moins de poids que les souris témoins $(146,7 \pm 8,2$ vs $174,7 \pm 7,5 \%$ du poids initial à 106 jours de régime, $n=4$, $p<0,05)$. De plus, les foies des souris SIC ont une masse plus faible que ceux des souris témoins, avec une quantité de glycogène identique mais une quantité en triglycérides beaucoup plus faible ( $25,6 \pm 4,6$ vs $88 \pm 17,2 \mathrm{mg} / \mathrm{g}$ de foie, $n=4, p<0,01$ ). Enfin, les souris SIC présentent une glycémie et une insulinémie à $6 \mathrm{~h}$ de jeûne plus faible que les souris témoins et montrent également une meilleure sensibilité à l'insuline.

La PIG exerce un effet protecteur contre le développement de l'obésité, du diabète et de la stéatose hépatique en améliorant notamment, l'homéostasie énergétique et la sensibilité à l'insuline.

Déclaration de liens d'intérêts Les auteurs n'ont pas précisé leurs éventuels liens d'intérêts.

http://dx.doi.org/10.1016/j.ando.2016.07.905

CO-062

NOV/CCN3 : une adipokine impliquée dans la résistance à l'insuline et la dépense énergétique

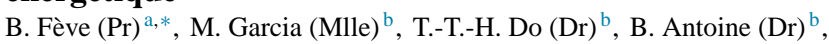
M. Moldes $(\mathrm{Dr})^{\mathrm{b}}$, G. Dorothée $(\mathrm{Dr})^{\mathrm{c}}$, C. Kazakian $(\mathrm{Mme})^{\mathrm{b}}$,

\footnotetext{
¿ Résumés présentés lors du $33^{\mathrm{e}}$ congrès de la société française d'endocrinologie Bordeaux 2016.
}

M. Auclair (Mme) $)^{\text {b }}$, M. Buyse (Dr) ${ }^{\mathrm{d}}$, T. Ledent (Mme) $)^{\mathrm{d}}$, P.-O. Marchal (Dr) ${ }^{\text {b }}$, M. Fesatidou (Mlle) ${ }^{\text {b }}$, A. Besseiche (Dr) ${ }^{\mathrm{e}}$,

H. Koseki (Dr $)^{\text {f }}$, S. Hiraoka $(D r)^{g}$, C. Chadjichristos $(D r)^{\text {h }}$, B. Blondeau (Dr) ${ }^{\mathrm{e}}$, R.-G. Denis (Dr) ${ }^{\mathrm{i}}$, S. Luquet $(\mathrm{Dr})^{\mathrm{i}}$, C. Martinerie $(\mathrm{Dr})^{\mathrm{b}}$

${ }^{a}$ Sorbonne universités, université Pierre et Marie Curie, Inserm, CdR Saint-Antoine UMR S938, hôpital Saint-Antoine, F-75012, et Assistance Publique des hôpitaux de Paris, service d'endocrinologie, hopital Saint-Antoine, IHU ICAN, Paris, France

${ }^{b}$ Sorbonne universités, université Pierre et Marie Curie, Inserm, CdR Saint-Antoine UMR S938 hopital Saint-Antoine, F-75012, IHU ICAN, Paris, France

${ }^{c}$ Sorbonne universités, université Pierre et Marie Curie, Inserm, CdR Saint-Antoine UMR S938 hopital Saint-Antoine, F-75012, Paris, France

${ }^{d}$ Sorbonne universités, université Pierre et Marie Curie, Inserm, CdR Saint-Antoine UMR S938 hôpital Saint-Antoine, F-75012, université Paris 11, IHU ICAN, Paris, France

${ }^{e}$ Sorbonne universités, université Pierre et Marie Curie, Inserm, CdR des Codeliers, IHU ICAN, Paris, France

${ }^{f}$ RIKEN Research Center for Allergy and Immunology (RCAI), RIKEN

Yokohama Institute, Yokohama, Japon

${ }^{g}$ Department of Biochemistry, Kobe Pharmaceutical University, Kobe, Japon

${ }^{h}$ Sorbonne universités, université Pierre et Marie Curie Paris 06, Inserm, hôpital Tenon, Paris, France

${ }^{i}$ Université Paris Diderot CNRS, BFA, Paris, France

* Auteur correspondant.

Adresse e-mail : bfeve@ free.fr (B. Fève)

L'identification de nouvelles adipokines faisant le lien entre obésité et insulinorésistance représente un enjeu majeur. Nous avons montré récemment que NOV/CCN3, une protéine matricellulaire multifonctionnelle est synthétisée et sécrétée par le tissu adipeux, avec des taux plasmatiques corrélés avec l'IMC. NOV a été antérieurement impliquée dans les processus de réparation tissulaire, la fibrose, les maladies inflammatoires et le cancer. Cependant, son rôle dans l'homéostasie énergétique restait inconnu. Nous avons ainsi étudié le phénotype de souris NOV-/- soumises à un régime normal ou hyperlipidique (HFD). De façon très nette, le poids des souris NOV-/- est nettement plus faible que celui des souris témoins, mais seulement en HFD. Ceci est lié à une réduction de masse grasse, avec des adipocytes plus petits, et une plus forte expression des acteurs de la thermogenèse au sein des dépôts adipeux blancs, et en particulier d'UCP1. Ceci va de pair avec une dépense énergétique accrue des souris invalidées. Les souris NOV-/- en HFD améliorent par ailleurs leur tolérance au glucose et leur sensibilité à l'insuline. De façon remarquable, les animaux NOV-/- ont un changement de phénotype macrophagique (M1 vers M2) et lymphocytaire dans leur tissu adipeux épididymaire, associé à une chute d'expression des cytokines proinflammatoires. Les animaux invalidés ont également une amélioration de leur signalisation insulinique in vivo. L'ensemble de ces données montre que NOV est une nouvelle adipokine impliquée dans la résistance à l'insuline associée à l'obésité. 